Article

\title{
(Non-)Thermal Production of WIMPs during Kination
}

\section{Luca Visinelli}

The Oskar Klein Centre for Cosmoparticle Physics, Department of Physics, Stockholm University, AlbaNova \& Nordita, KTH Royal Institute of Technology and Stockholm University, Roslagstullsbacken 23, 10691 Stockholm, Sweden

* Correspondence: luca.visinelli@fysik.su.se

\begin{abstract}
Understanding the nature of the Dark Matter (DM) is one of the current challenges in modern astrophysics and cosmology. Knowing the properties of the DM particle would shed light on physics beyond the Standard Model and even provide us with details of the early Universe. In fact, the detection of such a relic would bring us information from the pre-Big Bang Nucleosynthesis (BBN) period, an epoch from which we have no data, and could even hint at inflationary physics. In this work, we assume that the expansion rate of the Universe after inflationary is governed by the kinetic energy of a scalar field $\phi$, in the so-called "kination" model. We assume that the $\phi$ field decays into both radiation and DM particles, which we take to be Weakly Interacting Massive Particles (WIMPs). The present abundance of WIMPs is then fixed during the kination period through either a thermal "freeze-out" or "freeze-in" mechanism, or through a non-thermal process governed by the decay of $\phi$. We explore the parameter space of this theory with the requirement that the present WIMP abundance provides the correct DM relic budget. Requiring that BBN occurs during the standard cosmological scenario sets a limit on the temperature at which the kination period ends. Using this limit and assuming the WIMP has a mass $m_{\chi}=100 \mathrm{GeV}$, we obtain that the thermally-averaged WIMP annihilation cross section has to satisfy the constraints $3.5 \times 10^{-16} \mathrm{GeV}^{-2} \lesssim\langle\sigma v\rangle \lesssim 1.4 \times 10^{-5} \mathrm{GeV}^{-2}$ in order for having at least one of the production mechanism to yield the observed amount of DM. This result shows how the properties of the WIMP particle, if ever measured, can yield information on the pre-BBN content of the Universe.
\end{abstract}

\section{Keywords: Cosmology; Dark Matter; Early Universe}

\section{Introduction}

The existence of a Dark Matter (DM) component in the Universe has long been established [1, 2], with a Weakly Interacting Massive Particle (WIMP) being among the best motivated particle candidates [3,4]. In the simplest scenario of the early Universe, WIMPs of mass $m_{\chi}$ interact with the Standard Model (SM) particles at a sufficiently high rate so that the chemical equilibrium is attained. Owing to the expansion rate of the Universe, when the temperature falls below $T_{\text {chem }} \approx m_{\chi} / 20$ WIMPs chemically decouple from the plasma and "freeze-out" of the equilibrium distribution [5-12]. After freeze-out, the number of WIMPs in a comoving volume is fixed and the WIMP relic abundance is preserved to present day, assuming that there is no subsequent change in the entropy of the matter-radiation fluid. Coincidentally, the thermally-averaged WIMP annihilation cross section needed to explain the observed DM is of the same order of magnitude as that obtained for a process mediated by weakly interactions. For a WIMP of mass $m_{\chi}=100 \mathrm{GeV}$, the annihilation cross section that provides the observed amount of DM satisfies $\langle\sigma v\rangle_{\text {std }} \approx 2 \times 10^{-9} \mathrm{GeV}^{-2}$. WIMPs continue to exchange momentum through elastic collisions with the plasma even after chemical decoupling, until this second mechanism also becomes inefficient and WIMPs decouple kinetically at a temperature $T_{\mathrm{kd}}$. Typically, $T_{\mathrm{kd}}$ ranges between $10 \mathrm{MeV}$ and a few $\mathrm{GeV}$ [13]. 
Even when considering this thermal production in the standard cosmological scenario, many caveats allow to alter the predicted relic density. Besides co-annihilation $[14,15]$, annihilation into forbidden channels [14,16,17], a momentum- or spin-dependent cross section [18-20], or Sommerfeld enhancement [21-24], one possibility is that the annihilation cross section into the SM sector is so low that WIMPs never reach thermal equilibrium, effectively "freezing-in" to the present relic density. Examples include models of Feebly Interacting Massive Particles [25-27].

WIMPs might also be produced non-thermally, through the decay of a parent particle $\phi$ [28], whose existence is motivated by the post-inflationary reheating scenario. In facts, assume an early inflationary stage at an energy scale $H_{I}$ driven by one massive scalar field $\rho$ (the inflaton), of mass $m_{\rho}$. When slow-roll is violated, around $m_{\rho} \sim H_{I}$, inflation ends and the inflaton field reheats the Universe by decaying into lighter degrees of freedom $[29,30]$. We are not entering the details of the reheating mechanism here. In the standard picture, a radiation-dominated period begins as soon as the inflaton field has decayed and reheated the Universe.

However, in some reheating models the inflaton might also decay into one or more additional hypothetical fields, say for example a field $\phi$ of mass $m_{\phi}$ which comes to dominate the post-inflationary stage. The inclusion of a non-standard cosmology between the reheating epoch right after inflation and the standard radiation-dominated scenario is motivated in realistic models of inflation, and might sensibly alter the WIMP relic density. A non-standard period might have lasted for a considerable amount of time, namely since the end of inflation down to a temperature which, using considerations on the Big Bang Nucleosynthesis (BBN) mechanism [31-35], can be as low as $\sim 5 \mathrm{MeV}$. In these modified cosmologies, various properties of the WIMPs like their free-streaming velocity and the temperature at which the kinetic decoupling occurs have been investigated [36-39].

In the Low Temperature Reheat Scenario (LTRS) [40-43], $\phi$ is a massive modulus which drives an early matter-dominated epoch, eventually decaying into Standard Model particles and, possibly, WIMPs. In the pre-BBN LTRS, the thermal (both freeze-out and freeze-in) and non-thermal production of WIMPs have both been extensively studied [36,44-60].

In the Kination Scenario (KS) [61-66], $\phi$ is a "fast-rolling" field whose kinetic energy governs the expansion rate of the post-inflationary Universe, with an equation of state relating the pressure $p_{\phi}$ and energy density $\rho_{\phi}$ of the fluid as $p_{\phi}=\rho_{\phi}$. Owing to the scaling of the energy density in radiation with the scale factor $\rho_{R} \sim a^{-4}$, which is slower than the scaling of the energy density in the $\phi$ field $\rho_{\phi} \sim a^{-6}$, the contribution from the radiation energy density in determining the expansion rate eventually becomes more important than that from the $\phi$ field. When the $\phi$ field redshifts away, the standard radiation-dominated cosmology takes place. Since the scalar field $\phi$ dominates the expansion rate for some period, KS differs from the superWIMP model of Refs. [67,68]. Thermal production of WIMPs in the KS has been discussed in Refs. [65,69-73], and has recently been investigated in Ref. [74], in light of recent data. Ref. [75] discussed the "relentless" thermal freeze-out in models where the $\phi$ field has a pressure $p_{\phi}>\rho_{\phi} / 3$, thus including KS as an important sub-case. Ref. [76] discusses an intermediate model between LTRS and KS, in which a sub-dominant massive scalar field reheats the Universe during a kination period governed by an additional field.

In this paper, we assume that the $\phi$ field driving kination might decay into both radiation and WIMPs, with a decay rate $\Gamma_{\phi}$ and a branching ratio into WIMPs equal to $b$. Contrarily to previous KS models, kination ends when $\Gamma_{\phi}$ is equal to the expansion rate of the Universe, so when the $\phi$ field has decayed instead of being redshifted away. WIMP production proceeds by assuming that, before the Universe gets to be dominated by radiation at a temperature $T_{\text {kin }} \gtrsim 5 \mathrm{MeV}$, the KS occurs. In the following, the subscript "kin" labels a quantity evaluated at $T_{\text {kin. }}$. We consider the thermal freeze-out and freeze-in mechanisms of WIMP production, governed by the thermal-averaged annihilation cross section times velocity $\langle\sigma v\rangle$. We also include the non-thermal WIMP production from the decay of the $\phi$ field. We check that the WIMP population is always under-abundant with respect to other forms of energy. In summary, we show that in the model the present WIMP relic abundance can be reached 
where $M_{P 1}$ is the Planck mass. We assume that the mass of the $\phi$ field is larger than both $\epsilon_{\chi}$ and $b m_{\chi}$. In this limit, Eqs. (1)-(3) simplify as

$$
\begin{aligned}
\dot{\rho}_{\phi}+6 H \rho_{\phi} & =-\Gamma_{\phi} \rho_{\phi}, \\
\dot{\rho}_{R}+4 H \rho_{R} & =\Gamma_{\phi} \rho_{\phi}, \\
\dot{n}_{\chi}+3 H n_{\chi} & =\frac{b \Gamma_{\phi}}{m_{\phi}} \rho_{\phi}-\langle\sigma v\rangle\left(n_{\chi}^{2}-n_{\mathrm{EQ}}^{2}\right) .
\end{aligned}
$$

At early times $t \ll 1 / \Gamma_{\phi}$, the Boltzmann Eq. (6) and the Friedmann Eq. (5) predict that, during KS, the energy density of the $\phi$ field and time scale as $\rho_{\phi} \sim a^{-6}$ and $t \sim a^{3}$, respectively. Contrarily to what found in kination models with negligible decay rate, for which $a \sim 1 / T$ [63-66], in the model we study temperature depends on the scale factor as $T \propto \rho_{\phi}^{1 / 8} \propto a^{-3 / 4}$. In more details, Eq. (7) can be reformulated as a differential equation describing the evolution of the entropy per comoving volume $s=\left(p_{R}+\rho_{R}\right) / T$, as

$$
\frac{d s}{d t}+3 H s=\frac{\Gamma_{\phi}}{T} \rho_{\phi}
$$

As a consequence, entropy is not conserved in the model we consider because of the appearance of

through four different methods, namely the thermal $(b=0)$ or non-thermal $(b \neq 0)$ production, either with or without ever reaching chemical equilibrium, as occurs in the LTRS [50,51].

Here, $n_{\chi}$ is the WIMP number density, with a value $n_{\mathrm{EQ}}$ when in chemical equilibrium, $\rho_{R}$ is the energy density in radiation, and we defined the WIMP energy through $\rho_{\chi}=\epsilon_{\chi} n_{\chi}$. The equation of state relating the energy density and the pressure of the $\phi$ field is given by $\rho_{\phi}=p_{\phi}$, and translates into the term $6 \mathrm{H} \rho_{\phi}$ appearing in Eq. (1). At any time, the temperature $T$ is defined through the energy density in the relativistic component as $\rho_{R}=\alpha T^{4}$, with $\alpha=\pi^{2} g_{*}(T) / 30$ and where $g_{*}(T)$ is the number of relativistic degrees of freedom. We assume that WIMPs have $g$ degrees of freedom, so that the equilibrium distribution is given by $\left(E^{2}=p^{2}+m_{\chi}^{2}\right)$

$$
n_{\mathrm{EQ}}=\int f(p) \frac{g d^{3} p}{(2 \pi)^{3}}=\frac{g}{2 \pi^{2}} \int_{m_{\chi}}^{+\infty} \frac{\sqrt{E^{2}-m_{\chi}^{2}}}{e^{E / T}+1} E d E .
$$


We switch to the independent coordinates $x=m_{\phi} a$ and $\tau=\Gamma_{\phi} t$, while we write the dependent quantities in terms of the fields

$$
\Phi=A x^{6} \rho_{\phi} / m_{\phi}, \quad R=A x^{4} \rho_{R} / m_{\phi}, \quad X=A x^{3} n_{\chi} .
$$

Fixing the constant $A$ through the Friedmann equation,

$$
\mathcal{H}=\frac{1}{x} \frac{d x}{d \tau}=\frac{H}{\Gamma_{\phi}}=\frac{\sqrt{\Phi+R x^{2}+x^{3} X}}{x^{3}},
$$

we obtain that Eq. (5) is recovered when

$$
A=\frac{8 \pi m_{\phi}}{3 M_{\mathrm{Pl}}^{2} \Gamma_{\phi}^{2}} \equiv \frac{m_{\phi}}{\rho_{\mathrm{kin}}}
$$

where we have defined the temperature $T_{\text {kin }}$ in the instantaneous thermalization approximation, $\rho_{\text {kin }}=\rho_{R}\left(T_{\text {kin }}\right)$, so that $\Gamma_{\phi}=H\left(T_{\text {kin }}\right)$. With this definition, we rewrite the system of Eqs. (6)-(8) as

$$
\begin{aligned}
\Phi^{\prime} & =\frac{-x^{2} \Phi}{\sqrt{\Phi+x^{2} R+x^{3} X}}, \\
R^{\prime} & =\frac{\Phi}{\sqrt{\Phi+x^{2} R+x^{3} X}}, \\
X^{\prime} & =\frac{b \Phi-s\left(X^{2}-X_{\mathrm{EQ}}^{2}\right)}{x \sqrt{\Phi+x^{2} R+x^{3} X}},
\end{aligned}
$$

where $s=\langle\sigma v\rangle / \Gamma_{\phi} A=\rho_{\mathrm{kin}}\langle\sigma v\rangle / \Gamma_{\phi} m_{\phi}$. This dimensionless form of the system has never been shown in the literature, and can be easily extended to cosmologies other than KS. We assume that the relativistic species is always at equilibrium, so that temperature is related to $R$ as $T=T_{\text {kin }} R^{1 / 4} / x$. At chemical equilibrium, the quantity $X_{\mathrm{EQ}}=A x^{3} n_{\mathrm{EQ}}$ is then

$$
X_{\mathrm{EQ}}=\frac{g m_{\phi}}{\rho_{\mathrm{kin}}}\left(\frac{m_{\chi} T_{\mathrm{kin}} x R^{1 / 4}}{2 \pi}\right)^{3 / 2} \exp \left(\frac{-m_{\chi} x}{T_{\text {kin }} R^{1 / 4}}\right) .
$$

The set of Eqs. (13)-(16) possesses a scaling symmetry,

$$
x \rightarrow \beta x, \quad \Phi \rightarrow \beta^{6} \Phi \quad R \rightarrow \beta^{4} R \quad X \rightarrow \beta^{3} X,
$$

for any value of $\beta$, thanks to which the solution to the set of Boltzmann equations is independent on choice of the initial value $\Phi\left(x_{I}\right)=\Phi_{I}$ at $x=x_{I}[45,46]$. We fix the initial condition by requiring that the Hubble rate at $x=x_{I}$ be $H_{I}=\sqrt{8 \pi \rho_{I} / 3 M_{\mathrm{Pl}}^{2}}$, where $\rho_{I}$ is the value of the energy density in the inflaton field at the inflation scale. Indeed, assuming that $\rho_{R}\left(x_{I}\right) \ll \rho_{\phi}\left(x_{I}\right) \equiv \rho_{I}$ gives

$$
\Phi(x)=\Phi_{I} \equiv \frac{\rho_{I}}{\rho_{\text {kin }}} x_{I}^{6}, \quad R(x)=\sqrt{\Phi_{I}}\left(x-x_{I}\right) .
$$

Since the energy density $\rho_{\phi}$ during $\phi$-domination satisfies $\rho_{\phi} \sim a^{-6}$, the transition to the standard radiation-dominated scenario occurs when

$$
\rho_{\phi, I}\left(\frac{x_{I}}{x_{\text {kin }}}\right)^{6}=\rho_{\text {kin }} \equiv \alpha T_{\text {kin }}^{4} .
$$
purpose, we solve Eqs. (13) and (14) for $T_{\text {kin }}=0.1 \mathrm{GeV}$, fixing the masses $m_{\chi}=100 \mathrm{GeV}$ and $m_{\phi}=$ 


\section{0}

\section{We}

(b) $x<x_{\text {kin, }}$ while for $x>x_{\text {kin }}$ we obtain the scaling $\rho_{R} \sim a^{-4}$ for a
dashed vertical line marks the value of $x_{\text {kin }}$ solution to Eq. (19).

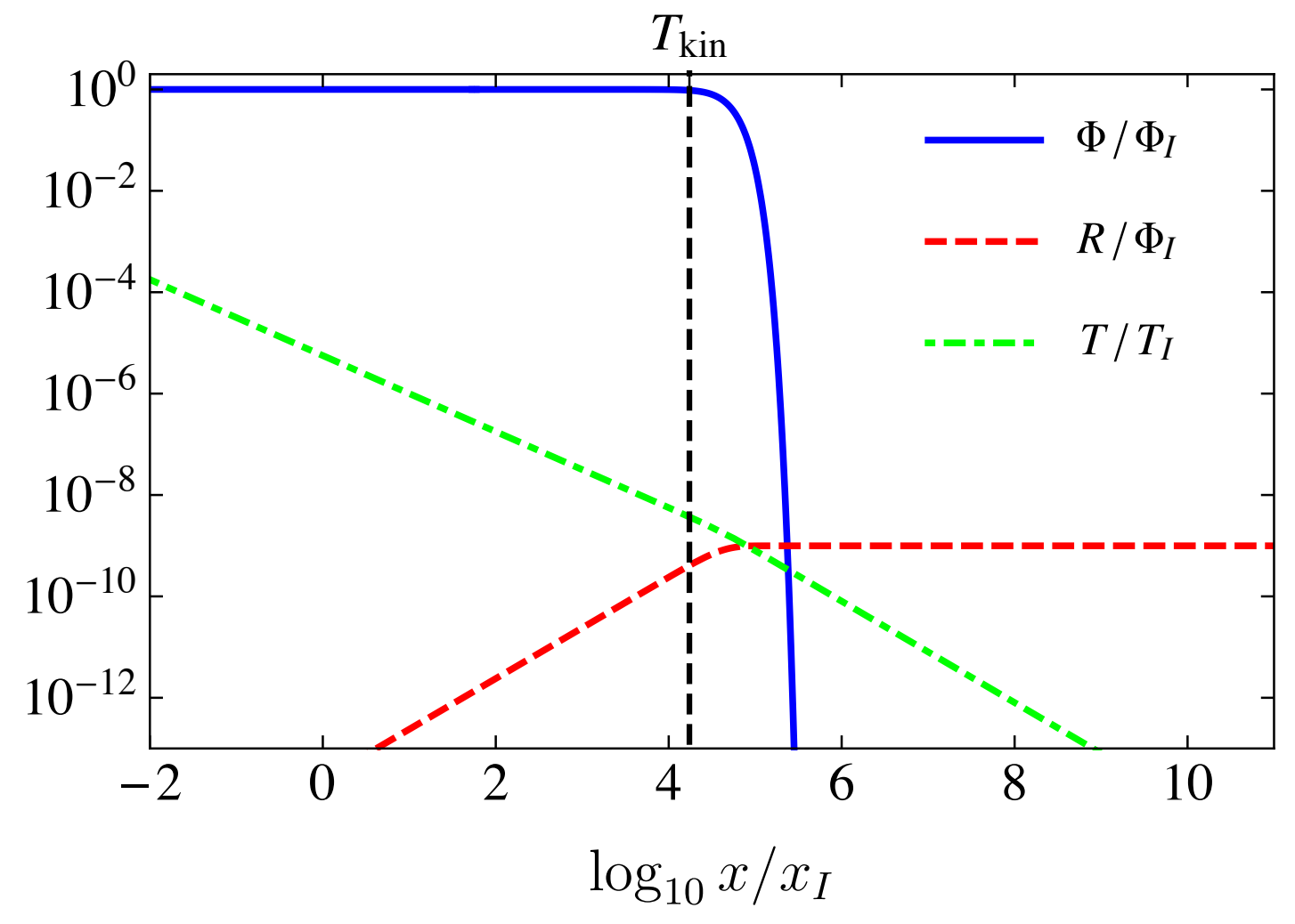

Figure 1. The quantities $\Phi$ and $R$, defined in Eq. (10) and related to $\rho_{\phi}$ and $\rho_{R}$ respectively, in units of the initial value $\Phi_{I}$. The vertical dashed line marks the moment at which the transition to the standard scenario occurs, according to Eq. (19). The green dot-dashed line shows the temperature $T(x)$, in units of its initial value $T\left(x_{I}\right)$.

$1000 \mathrm{TeV}$ and assuming that we can safely neglect the contribution of WIMPs to the total energy density. We have plot $\Phi$ (blue solid line) and $R$ (red dashed line) in units of $\Phi_{I}$, as well as the temperature $T / T_{I}$ (black dot-dashed line). The behavior of $\Phi$ confirms the scaling $\rho_{\phi} \sim a^{-6}$ and $\rho_{R} \sim a^{-3}$ at early times

\section{Production of WIMPs during kination}

With this framework, we solve the set of Boltzmann Eqs. (13)-(15) for different values of $T_{\text {kin }}$, to obtain the WIMP relic abundance $n_{\text {kin }} \equiv n_{\chi}\left(T_{\text {kin }}\right)$ when the $\phi$ field decays, after which the WIMP number density in a comoving volume is fixed. The present WIMP energy density in units of the critical density $\rho_{c}$ is then

$$
\Omega_{\chi}=\frac{m_{\chi}}{m_{\phi}} \frac{\rho_{\mathrm{kin}}}{\rho_{c}} \frac{g_{S}\left(T_{0}\right)}{g_{S}\left(T_{\mathrm{kin}}\right)}\left(\frac{T_{0}}{T_{\mathrm{kin}}}\right)^{3} \frac{X_{\mathrm{kin}}}{\sqrt{\Phi_{I}}}
$$

where $X_{\text {kin }}=A x_{\text {kin }}^{3} n_{\text {kin }}, T_{0}$ is the present temperature of the radiation bath and $g_{S}(T)$ is the number of entropy degrees of freedom at temperature $T$. We show different values of the abundance $\Omega_{\chi} h^{2}$ in Fig. 2, as a function of the temperature $T_{\text {kin }}$, the annihilation cross section $\langle\sigma v\rangle$, and the branching ratio $b$. We have used three different values of $b=10^{-10}, 10^{-5}$, and $10^{-1}$, as well as five different values of $\langle\sigma v\rangle$. The value $\langle\sigma v\rangle=\langle\sigma v\rangle_{\text {std }}$ gives the correct amount of DM when $T_{\text {kin }} \gtrsim m_{\chi} / 20$, since in such scenario the chemical decoupling of WIMPs occurs in the standard cosmology. The extra dashed line at $T_{\text {kin }}=0.1 \mathrm{GeV}$ tracks the specific solution of the Boltzmann equations later used in Fig. 3. The value 


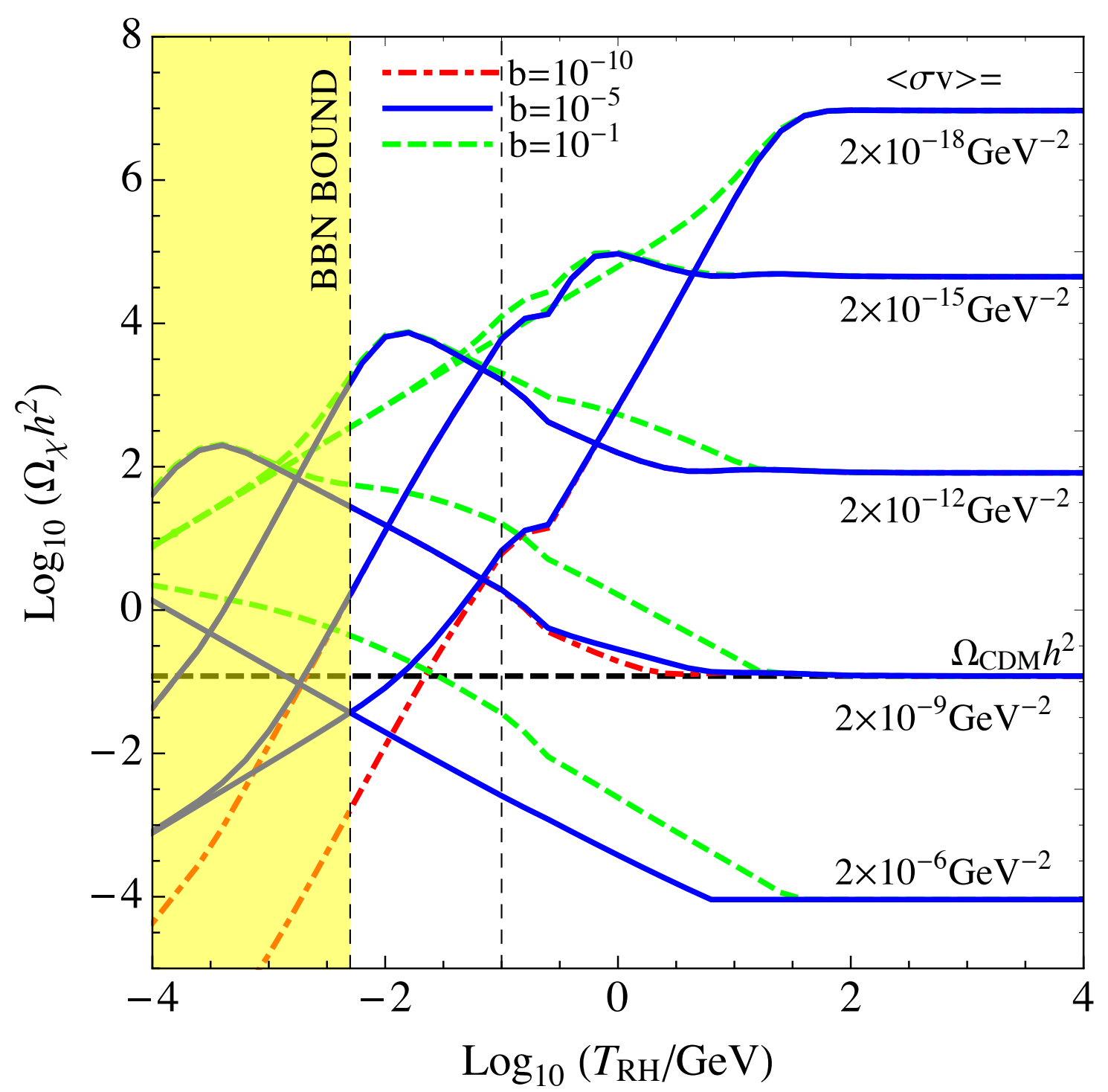

Figure 2. The present WIMP relic abundance for different values of $\langle\sigma v\rangle$ (see figure labels), and for different values of the branching ratio: $b=10^{-10}$ (red dot-dashed line), $b=10^{-5}$ (blue solid line), and $b=10^{-1}$ (green dashed line), as a function of $T_{\text {kin }}$. The horizontal dashed line shows the measured dark matter abundance $\Omega_{\mathrm{DM}} h^{2} \sim 0.12$. The vertical yellow band defines the region excluded by BBN considerations, $T_{\text {kin }} \geq 5 \mathrm{MeV}$. The vertical line at $T_{\text {kin }}=0.1 \mathrm{GeV}$ marks the solutions to the Boltzmann Eq. (15) later used for Fig. 3, using $b=0.1$.

of $n_{\text {kin }}$ depends on the specific mechanism that dominates the production of the WIMP population. According to the values of $b$ and $\langle\sigma v\rangle$, four possibilities appear, namely:

- Thermal production with chemical equilibrium ("freeze-out", Mechanism 1). We first focus on the case $b=0$, corresponding to the negligible decay with respect to the annihilation into standard model particles and approximated by the red dot-dashed and blue solid lines in Fig. 2. 
We assume that the temperature $T_{\text {kin }}$ is lower than the freeze-out temperature $T_{\text {f.o }}$, which is defined as the temperature at which $n\langle\sigma v\rangle=H$, or

$$
n_{\mathrm{EQ}}\left(T_{\mathrm{f} . \mathrm{o}}\right)=\left(\frac{T_{\text {f.o }}}{T_{\text {kin }}}\right)^{4} \frac{H\left(T_{\text {kin }}\right)}{\langle\sigma v\rangle} .
$$

However, contrary to what obtained in the standard radiation-dominated scenario, the WIMP number density in the kination cosmology is not fixed at $T_{\text {f.o }}$ and annihilation continues until the temperature drops to $T_{\text {kin }}$ and the expansion rate transitions to that of a radiation-dominated one [75]. We assume that the freeze-out is reached at $x_{\text {f.o. }}$ for which $X\left(x_{\text {f.o. }}\right)=X_{\text {f.o. }}$. For later times, using the approximation in Eq. (19), Eq. (15) reduces to

$$
X^{\prime}=-\frac{\rho_{\text {kin }}\langle\sigma v\rangle}{\Gamma_{\phi} m_{\phi}} \frac{X^{2}}{x \sqrt{\Phi_{I}}}
$$

whose solution at $x>x_{\text {f.o. }}$ give the abundance of thermally produced WIMPs at $T_{\text {kin }}$, which reads

$$
X_{\text {kin,th }}=\left[\frac{1}{X_{\text {f.o. }}}+\frac{\langle\sigma v\rangle T_{\text {kin }}^{2}}{\sqrt{\Phi_{I}}} \frac{M_{\mathrm{Pl}}}{m_{\phi}} \ln \frac{x_{\text {kin }}}{x_{\mathrm{fo}}}\right]^{-1} .
$$

Neglecting $X_{\text {f.o., }}$ the present abundance in Eq. (20) for the standard freeze-out mechanism gives

$$
\Omega_{\chi}=\frac{g_{S}\left(T_{0}\right)}{g_{S}\left(T_{\text {kin }}\right)} \frac{m_{\chi}}{M_{\mathrm{Pl}}} \frac{\rho_{\text {kin }}}{\rho_{c}} \frac{T_{0}^{3}}{\langle\sigma v\rangle T_{\text {kin }}^{5}}\left(\ln \frac{x_{\text {kin }}}{x_{\text {fo }}}\right)^{-1} \propto \frac{1}{T_{\text {kin }}} .
$$

The solution describes the lines with negative slopes in Fig. 2, for $b=10^{-10}$ and $b=10^{-5}$ and for the cross sections $\langle\sigma v\rangle=2 \times 10^{-6} \mathrm{GeV}^{-2},\langle\sigma v\rangle=2 \times 10^{-9} \mathrm{GeV}^{-2}$, and $\langle\sigma v\rangle=2 \times 10^{-12} \mathrm{GeV}^{-2}$.

- Thermal production without ever reaching chemical equilibrium ("freeze-in", Mechanism 2). If the cross section is sufficiently low [74], WIMPs never reach thermal equilibrium and their number density freezes in at a fixed quantity. Since the number density of particles is always smaller than their value at thermal equilibrium, we neglect $X \ll X_{\mathrm{EQ}}$ so Eq. (15) with $b=0$ reads

$$
X^{\prime}=\frac{\rho_{\text {kin }}\langle\sigma v\rangle}{\Gamma_{\phi} m_{\phi}} \frac{\left(X_{\mathrm{EQ}}^{2}\right)}{x \sqrt{\Phi_{I}}}=c_{1} x^{11 / 4} \exp \left(-2 c_{2} x^{3 / 4}\right),
$$

where

$$
c_{1}=\frac{g^{2}\langle\sigma v\rangle m_{\phi} m_{\chi}^{3} T_{\mathrm{kin}}^{3}}{(2 \pi)^{3} \Gamma_{\phi} \rho_{\mathrm{kin}} \Phi_{I}^{1 / 8}}, \quad \text { and } \quad c_{2}=\frac{m_{\chi}}{T_{\mathrm{kin}} \Phi_{I}^{1 / 8}} .
$$

The solution to Eq. (25) reaches the asymptotic value of $X$ at freeze-in

$$
X_{\mathrm{kin}, \mathrm{fi}}=\frac{c_{1}}{c_{2}^{5}}=\frac{g^{2}\langle\sigma v\rangle m_{\phi} T_{\mathrm{kin}}^{8} \sqrt{\Phi_{I}}}{(2 \pi)^{3} \Gamma_{\phi} \rho_{\mathrm{kin}} m_{\chi}^{2}}
$$

which is reached when $x_{\mathrm{fi}}=\left(6 / 11 c_{2}\right)^{4 / 3}$. The present abundance is then

$$
\Omega_{\chi}=\frac{g_{S}\left(T_{0}\right)}{g_{S}\left(T_{\text {kin }}\right)} \frac{g^{2}\langle\sigma v\rangle T_{0}^{3} T_{\text {kin }}^{5}}{(2 \pi)^{3} \Gamma_{\phi} \rho_{c} m_{\chi}} \propto T_{\text {kin }}^{3} .
$$

The solution describes the lines with positive slopes in Fig. 2, for $b=10^{-10}$ and $b=10^{-5}$ and for the cross sections $\langle\sigma v\rangle=2 \times 10^{-12} \mathrm{GeV}^{-2},\langle\sigma v\rangle=2 \times 10^{-15} \mathrm{GeV}^{-2}$, and $\langle\sigma v\rangle=$ $2 \times 10^{-18} \mathrm{GeV}^{-2}$.

- Non-thermal production without chemical equilibrium (Mechanism 3). We now discuss the non-thermal production of dark matter, in the case in which the particle has never reached 
the chemical equilibrium. For a large branching ratio $b=O(0.1)$, and for $T_{\text {kin }} \ll m_{\chi}$, the abundance of dark matter is set by the decay of the $\phi$ field, with a number density at $T_{\text {kin }}$ given by $[50,52,55,58,60,77]$

$$
n_{\text {kin }} \approx b n_{\phi}\left(T_{\text {kin }}\right)=b \frac{\rho_{\text {kin }}}{m_{\phi}} .
$$

Deriving the result from directly integrating Eq. (15) with $\langle\sigma v\rangle=0$ and neglecting the contributions from $R$ and $X$ in the denominator gives an extra logarithmic dependence on $x_{\text {kin, }}$ as

$$
X_{\text {kin,decay }}=b \sqrt{\Phi_{I}} \ln \frac{x_{\text {kin }}}{x_{I}} .
$$

\section{Discussion and summary}

In Fig. 3, we summarize the results obtained by considering the behavior of the quantity $X$ solution to the Boltzmann Eq. (15) for $b=0.1$, the green dot-dashed line in Fig. 2, and for $T_{\text {kin }}=0.1 \mathrm{GeV}$. In facts, for this choice of the parameters, the different values of $\langle\sigma v\rangle$ in Fig. 2 give the different production mechanisms discussed. In Fig. 3, the black solid line represents $X_{\mathrm{EQ}}$, while the different mechanisms of productions are all given for different values of $\langle\sigma v\rangle$, with $\langle\sigma v\rangle=2 \times 10^{-9} \mathrm{GeV}^{-2}$ (blue line) describing non-thermal production with chemical equilibrium (Mechanism 4), $\langle\sigma v\rangle=2 \times 10^{-18} \mathrm{GeV}^{-2}$ (green line) describing non-thermal production without chemical equilibrium (Mechanism 3), $\langle\sigma v\rangle=2 \times$ $10^{-15} \mathrm{GeV}^{-2}$ (orange line) describing thermal production without chemical equilibrium (Mechanism 2), and $\langle\sigma v\rangle=2 \times 10^{-12} \mathrm{GeV}^{-2}$ (red line) describing thermal production with chemical equilibrium 
(Mechanism 1). In addition to the thermal mechanisms of production recently discussed in Refs. [74,75],

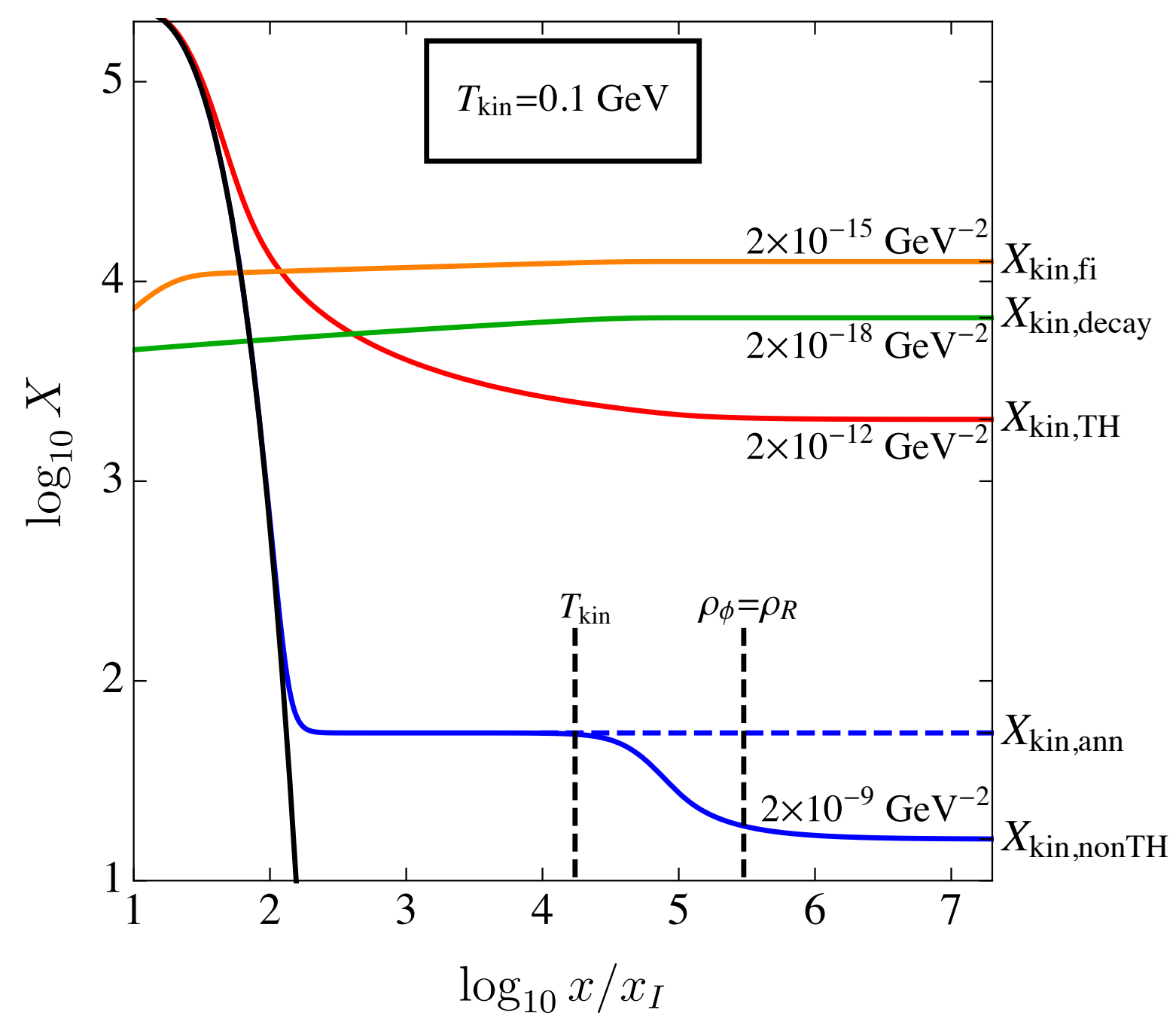

Figure 3. The solution to the Boltzmann Eq. (15) for different values of the annihilation cross section. We have set $T_{\text {kin }}=0.1 \mathrm{GeV}$ and, when non-thermal production is considered, $b=0.1$. See text for further details.

we have included the possibility that the field $\phi$ responsible for the kination period decays into radiation and WIMPs. We have then studied the non-thermal production of WIMPs in the kination cosmology, as summarized in Fig. 2. Given the value $\langle\sigma v\rangle_{\text {std }}=2 \times 10^{-9} \mathrm{GeV}^{-2}$ that gives the present abundance of DM from the freeze-out of WIMPs in the standard cosmology, Fig. 2 shows that larger values of $\langle\sigma v\rangle$ can still lead to the right DM abundance, if WIMPs are produced either through Mechanisms 1) or 4) during the KS. Similarly, we can have $\langle\sigma v\rangle$ smaller than its standard value and still have the correct amount of DM, if WIMPs are produced through Mechanism 2). Non-thermal production without chemical equilibrium (Mechanism 3) would lead to the correct amount of DM only for values of $T_{\text {kin }}$ that are excluded by the BBN considerations. Using the bound $T_{\text {kin }} \gtrsim 5 \mathrm{MeV}$, we infer the possible range of the parameter

$$
3.5 \times 10^{-16} \mathrm{GeV}^{-2} \lesssim\langle\sigma v\rangle \lesssim 1.4 \times 10^{-5} \mathrm{GeV}^{-2}
$$


the lower bound being obtained by using Mechanism 2 and the upper bound being given by Mechanism 4 with $b=1$. This result is valid for a WIMP of mass $m_{\chi}=100 \mathrm{GeV}$ and $m_{\phi}=1000 \mathrm{TeV}$.

To summarize, if the DM is a WIMP of mass $m_{\chi}=100 \mathrm{GeV}$, and if the annihilation cross section is measured to lie outside of the bound in Eq. (35), then the kination model discussed would have to be discarded. The same analysis can be performed by varying the masses of the WIMP and of the $\phi$ field, which would lead to different values of the bounds in Eq. (35). If ever discovered, the properties of the WIMP could then shed light on the pre-BBN cosmology.

Acknowledgments: We thank Adrienne Erickcek, Kayla Redmond, and Sunny Vagnozzi for reading earlier versions of the manuscript. We acknowledge support by the Vetenskapsrådet (Swedish Research Council) through contract No. 638-2013-8993 and the Oskar Klein Centre for Cosmoparticle Physics.

Conflicts of Interest: The authors declare no conflict of interest. The founding sponsors had no role in the design of the study; in the collection, analyses, or interpretation of data; in the writing of the manuscript, and in the decision to publish the results.

\section{Abbreviations}

The following abbreviations are used in this manuscript:

DM Dark Matter

BBN Big Bang Nucleosynthesis

WIMP Weakly Interacting Massive Particle

LTRS Low Temperature Reheat Scenario

KS Kination Scenario

1. Ade, P.A.R.; others. Joint Analysis of BICEP2/Keck Array and Planck Data. Phys. Rev. Lett. 2015, 114, 101301, [arXiv:astro-ph.CO/1502.00612].

2. Ade, P.A.R.; others. Planck 2015 results. XIII. Cosmological parameters. Astron. Astrophys. 2016, 594, A13, [arXiv:astro-ph.CO/1502.01589].

3. Jungman, G.; Kamionkowski, M.; Griest, K. Supersymmetric dark matter. Phys. Rept. 1996, 267, 195-373, [arXiv:hep-ph/hep-ph/9506380].

4. Bertone, G.; Hooper, D.; Silk, J. Particle dark matter: Evidence, candidates and constraints. Phys. Rept. 2005, 405, 279-390, [arXiv:hep-ph/hep-ph/0404175].

5. Vysotsky, M.I.; Dolgov, A.D.; Zeldovich, Ya.B. Cosmological Restriction on Neutral Lepton Masses. JETP Lett. 1977, 26, 188-190. [Pisma Zh. Eksp. Teor. Fiz.26,200(1977)].

6. Hut, P. Limits on Masses and Number of Neutral Weakly Interacting Particles. Phys. Lett. 1977, 69 B, 85.

7. Sato, K.; Kobayashi, M. Cosmological Constraints on the Mass and the Number of Heavy Lepton Neutrinos. Prog. Theor. Phys. 1977, 58, 1775.

8. Lee, B.W.; Weinberg, S. Cosmological Lower Bound on Heavy Neutrino Masses. Phys. Rev. Lett. 1977, 39, 165-168.

9. Dicus, D.A.; Kolb, E.W.; Teplitz, V.L. Cosmological Implications of Massive, Unstable Neutrinos: New and Improved. Astrophys. J. 1978, 221, 327-341.

10. Steigman, G. Cosmology Confronts Particle Physics. Ann. Rev. Nucl. Part. Sci. 1979, 29, 313-338.

11. Bernstein, J.; Brown, L.S.; Feinberg, G. The Cosmological Heavy Neutrino Problem Revisited. Phys. Rev. 1985, D32, 3261.

12. Kolb, E.W.; Olive, K.A. The Lee-Weinberg Bound Revisited. Phys. Rev. 1986, D33, 1202. [Erratum: Phys. Rev.D34,2531(1986)].

13. Profumo, S.; Sigurdson, K.; Kamionkowski, M. What mass are the smallest protohalos? Phys. Rev. Lett. 2006, 97, 031301, [arXiv:astro-ph/astro-ph/0603373].

14. Griest, K.; Seckel, D. Three exceptions in the calculation of relic abundances. Phys. Rev. 1991, D43, 3191-3203. 
15. Edsjo, J.; Gondolo, P. Neutralino relic density including coannihilations. Phys. Rev. 1997, D56, 1879-1894, [arXiv:hep-ph/hep-ph/9704361].

16. D'Agnolo, R.T.; Ruderman, J.T. Light Dark Matter from Forbidden Channels. Phys. Rev. Lett. 2015, 115, 061301, [arXiv:hep-ph/1505.07107].

17. Cline, J.; Liu, H.; Slatyer, T.; Xue, W. Enabling Forbidden Dark Matter. Phys. Rev. 2017, D96, 083521, [arXiv:hep-ph/1702.07716].

18. Chang, S.; Pierce, A.; Weiner, N. Momentum Dependent Dark Matter Scattering. JCAP 2010, 1001, 006, [arXiv:hep-ph/0908.3192].

19. Fan, J.; Reece, M.; Wang, L.T. Non-relativistic effective theory of dark matter direct detection. JCAP 2010, 1011, 042, [arXiv:hep-ph/1008.1591].

20. Fitzpatrick, A.L.; Haxton, W.; Katz, E.; Lubbers, N.; Xu, Y. The Effective Field Theory of Dark Matter Direct Detection. JCAP 2013, 1302, 004, [arXiv:hep-ph/1203.3542].

21. Cirelli, M.; Kadastik, M.; Raidal, M.; Strumia, A. Model-independent implications of the e+-, anti-proton cosmic ray spectra on properties of Dark Matter. Nucl. Phys. 2009, B813, 1-21, [arXiv:hep-ph/0809.2409]. [Addendum: Nucl. Phys.B873,530(2013)].

22. Arkani-Hamed, N.; Finkbeiner, D.P.; Slatyer, T.R.; Weiner, N. A Theory of Dark Matter. Phys. Rev. 2009, D79, 015014, [arXiv:hep-ph/0810.0713].

23. Pospelov, M.; Ritz, A. Astrophysical Signatures of Secluded Dark Matter. Phys. Lett. 2009, B671, 391-397, [arXiv:hep-ph/0810.1502].

24. Fox, P.J.; Poppitz, E. Leptophilic Dark Matter. Phys. Rev. 2009, D79, 083528, [arXiv:hep-ph/0811.0399].

25. Hall, L.J.; Jedamzik, K.; March-Russell, J.; West, S.M. Freeze-In Production of FIMP Dark Matter. JHEP 2010, 03, 080, [arXiv:hep-ph/0911.1120].

26. Co, R.T.; D’Eramo, F.; Hall, L.J.; Pappadopulo, D. Freeze-In Dark Matter with Displaced Signatures at Colliders. JCAP 2015, 1512, 024, [arXiv:hep-ph/1506.07532].

27. Bernal, N.; Heikinheimo, M.; Tenkanen, T.; Tuominen, K.; Vaskonen, V. The Dawn of FIMP Dark Matter: A Review of Models and Constraints. Int. J. Mod. Phys. 2017, A32, 1730023, [arXiv:hep-ph/1706.07442].

28. Kamionkowski, M.; Turner, M.S. Thermal relics: Do we know their abundances? Phys. Rev. D 1990, 42, 3310-3320

29. Kofman, L.; Linde, A.D.; Starobinsky, A.A. Reheating after inflation. Phys. Rev. Lett. 1994, 73, 3195-3198, [arXiv:hep-th/hep-th/9405187].

30. Kofman, L.; Linde, A.D.; Starobinsky, A.A. Towards the theory of reheating after inflation. Phys. Rev. 1997, D56, 3258-3295, [arXiv:hep-ph/hep-ph/9704452].

31. Kawasaki, M.; Kohri, K.; Sugiyama, N. Cosmological constraints on late time entropy production. Phys. Rev. Lett. 1999, 82, 4168, [arXiv:astro-ph/astro-ph/9811437].

32. Kawasaki, M.; Kohri, K.; Sugiyama, N. MeV scale reheating temperature and thermalization of neutrino background. Phys. Rev. 2000, D62, 023506, [arXiv:astro-ph/astro-ph/0002127].

33. Hannestad, S. What is the lowest possible reheating temperature? Phys. Rev. 2004, D70, 043506, [arXiv:astro-ph/astro-ph/0403291].

34. Ichikawa, K.; Kawasaki, M.; Takahashi, F. The Oscillation effects on thermalization of the neutrinos in the Universe with low reheating temperature. Phys. Rev. 2005, D72, 043522, [arXiv:astro-ph/astro-ph/0505395].

35. De Bernardis, F.; Pagano, L.; Melchiorri, A. New constraints on the reheating temperature of the universe after WMAP-5. Astropart. Phys. 2008, 30, 192-195.

36. Gelmini, G.B.; Gondolo, P. Ultra-cold WIMPs: relics of non-standard pre-BBN cosmologies. JCAP 2008, 0810, 002, [arXiv:astro-ph/0803.2349].

37. Visinelli, L.; Gondolo, P. Kinetic decoupling of WIMPs: analytic expressions. Phys. Rev. 2015, D91, 083526, [arXiv:astro-ph.CO/1501.02233].

38. Waldstein, I.R.; Erickcek, A.L.; Ilie, C. Quasidecoupled state for dark matter in nonstandard thermal histories. Phys. Rev. 2017, D95, 123531, [arXiv:astro-ph.CO/1609.05927].

39. Waldstein, I.R.; Erickcek, A.L. Comment on "Kinetic decoupling of WIMPs: Analytic expressions". Phys. Rev. 2017, D95, 088301, [arXiv:astro-ph.CO/1707.03417].

40. Dine, M.; Fischler, W. The Not So Harmless Axion. Phys. Lett. 1983, 120B, 137-141.

41. Steinhardt, P.J.; Turner, M.S. Saving the Invisible Axion. Phys. Lett. 1983, 129B, 51. 
42. Turner, M.S. Coherent Scalar Field Oscillations in an Expanding Universe. Phys. Rev. 1983, D28, 1243.

43. Scherrer, R.J.; Turner, M.S. Decaying Particles Do Not Heat Up the Universe. Phys. Rev. 1985, D31, 681.

44. Lyth, D.H.; Stewart, E.D. Thermal inflation and the moduli problem. Phys. Rev. 1996, D53, 1784-1798, [arXiv:hep-ph/hep-ph/9510204].

45. Chung, D.J.H.; Kolb, E.W.; Riotto, A. Production of massive particles during reheating. Phys. Rev. 1999, D60, 063504, [arXiv:hep-ph/hep-ph/9809453].

46. Giudice, G.F.; Kolb, E.W.; Riotto, A. Largest temperature of the radiation era and its cosmological implications. Phys. Rev. 2001, D64, 023508, [arXiv:hep-ph/hep-ph/0005123].

47. Moroi, T.; Randall, L. Wino cold dark matter from anomaly mediated SUSY breaking. Nuclear Physics $B$ 2000, 570, 455-472, [hep-ph/9906527].

48. Fujii, M.; Hamaguchi, K. Nonthermal dark matter via Affleck-Dine baryogenesis and its detection possibility. Phys. Rev. 2002, D66, 083501, [arXiv:hep-ph/hep-ph/0205044].

49. Fujii, M.; Ibe, M.; Yanagida, T. Thermal leptogenesis and gauge mediation. Phys. Rev. 2004, D69, 015006, [arXiv:hep-ph/hep-ph/0309064].

50. Gelmini, G.B.; Gondolo, P. Neutralino with the right cold dark matter abundance in (almost) any supersymmetric model. Phys. Rev. 2006, D74, 023510, [arXiv:hep-ph/hep-ph/0602230].

51. Gelmini, G.; Gondolo, P.; Soldatenko, A.; Yaguna, C.E. The Effect of a late decaying scalar on the neutralino relic density. Phys. Rev. 2006, D74, 083514, [arXiv:hep-ph/hep-ph/0605016].

52. Acharya, B.S.; Kane, G.; Watson, S.; Kumar, P. A Non-thermal WIMP Miracle. Phys. Rev. 2009, D80, 083529, [arXiv:astro-ph.CO/0908.2430].

53. Grin, D.; Smith, T.; Kamionkowski, M. Thermal axion constraints in non-standard thermal histories. AIP Conf. Proc. 2010, 1274, 78-84.

54. Harigaya, K.; Kawasaki, M.; Mukaida, K.; Yamada, M. Dark Matter Production in Late Time Reheating. Phys. Rev. 2014, D89, 083532, [arXiv:hep-ph/1402.2846].

55. Baer, H.; Choi, K.Y.; Kim, J.E.; Roszkowski, L. Dark matter production in the early Universe: beyond the thermal WIMP paradigm. Phys. Rept. 2015, 555, 1-60, [arXiv:hep-ph/1407.0017].

56. Monteux, A.; Shin, C.S. Thermal Goldstino Production with Low Reheating Temperatures. Phys. Rev. 2015, D92, 035002, [arXiv:hep-ph/1505.03149].

57. Reece, M.; Roxlo, T. Nonthermal production of dark radiation and dark matter. JHEP 2016, 09, 096, [arXiv:hep-ph/1511.06768].

58. Kane, G.L.; Kumar, P.; Nelson, B.D.; Zheng, B. Dark matter production mechanisms with a nonthermal cosmological history: A classification. Phys. Rev. 2016, D93, 063527, [arXiv:hep-ph/1502.05406].

59. Erickcek, A.L. The Dark Matter Annihilation Boost from Low-Temperature Reheating. Phys. Rev. 2015, D92, 103505, [arXiv:astro-ph.CO/1504.03335].

60. Kim, H.; Hong, J.P.; Shin, C.S. A map of the non-thermal WIMP. Phys. Lett. 2017, B768, 292-298, [arXiv:hep-ph/1611.02287].

61. Barrow, J.D. Massive Particles as a Probe of the Early Universe. Nucl. Phys. 1982, B208, 501.

62. Ford, L.H. Gravitational Particle Creation and Inflation. Phys. Rev. 1987, D35, 2955.

63. Spokoiny, B. Deflationary universe scenario. Phys. Lett. 1993, B315, 40-45, [arXiv:gr-qc/gr-qc/9306008].

64. Joyce, M. Electroweak Baryogenesis and the Expansion Rate of the Universe. Phys. Rev. 1997, D55, 1875-1878, [arXiv:hep-ph/hep-ph/9606223].

65. Salati, P. Quintessence and the relic density of neutralinos. Phys. Lett. 2003, B571, 121-131, [arXiv:astro-ph/astro-ph/0207396].

66. Profumo, S.; Ullio, P. SUSY dark matter and quintessence. JCAP 2003, 0311, 006, [arXiv:hep-ph/hep-ph/0309220].

67. Feng, J.L.; Rajaraman, A.; Takayama, F. SuperWIMP dark matter signals from the early universe. Phys. Rev. 2003, D68, 063504, [arXiv:hep-ph/hep-ph/0306024].

68. Feng, J.L.; Su, S.f.; Takayama, F. SuperWIMP gravitino dark matter from slepton and sneutrino decays. Phys. Rev. 2004, D70, 063514, [arXiv:hep-ph/hep-ph/0404198].

69. Pallis, C. Quintessential kination and cold dark matter abundance. JCAP 2005, 0510, 015, [arXiv:hep-ph/hep-ph/0503080].

70. Gomez, M.E.; Lola, S.; Pallis, C.; Rodriguez-Quintero, J. Quintessential Kination and Thermal Production of SUSY e-WIMPs. AIP Conf. Proc. 2009, 1115, 157-162, [arXiv:hep-ph/0809.1982]. 
71. Lola, S.; Pallis, C.; Tzelati, E. Tracking Quintessence and Cold Dark Matter Candidates. JCAP 2009, 0911, 017, [arXiv:hep-ph/0907.2941].

72. Lewicki, M.; Rindler-Daller, T.; Wells, J.D. Enabling Electroweak Baryogenesis through Dark Matter. JHEP 2016, 06, 055, [arXiv:hep-ph/1601.01681].

73. Artymowski, M.; Lewicki, M.; Wells, J.D. Gravitational wave and collider implications of electroweak baryogenesis aided by non-standard cosmology. JHEP 2017, 03, 066, [arXiv:hep-ph/1609.07143].

74. Redmond, K.; Erickcek, A.L. New Constraints on Dark Matter Production during Kination. Phys. Rev. 2017, D96, 043511, [arXiv:hep-ph/1704.01056].

75. D'Eramo, F.; Fernandez, N.; Profumo, S. When the Universe Expands Too Fast: Relentless Dark Matter 2017. [arXiv:hep-ph/1703.04793].

76. Pallis, C. Kination-dominated reheating and cold dark matter abundance. Nucl. Phys. 2006, B751, 129-159, [arXiv:hep-ph/hep-ph/0510234].

77. Choi, K.Y.; Kim, J.E.; Lee, H.M.; Seto, O. Neutralino dark matter from heavy axino decay. Phys. Rev. 2008, D77, 123501, [arXiv:hep-ph/0801.0491]. 